\title{
An extension of a variant of d'Alemberts functional equation on compact groups
}

\section{Iz-iddine EL-Fassi}

Department of Mathematics,

Faculty of Sciences,

Ibn Tofail University, Morocco

email: izidd-math@hotmail.fr
Abdellatif Chahbi

Department of Mathematics,

Faculty of Sciences,

Ibn Tofail University, Morocco

email: abdellatifchahbi@gmail.com

\section{Samir Kabbaj \\ Department of Mathematics, \\ Faculty of Sciences, \\ Ibn Tofail University, Morocco \\ email: samkabbaj@yahoo.fr}

Abstract. All paper is related with the non-zero continuous solutions $f: G \rightarrow \mathbb{C}$ of the functional equation

$$
f(x \sigma(y))+f(\tau(y) x)=2 f(x) f(y), \quad x, y \in G,
$$

where $\sigma, \tau$ are continuous automorphism or continuous anti-automorphism defined on a compact group $G$ and possibly non-abelian, such that $\sigma^{2}=$ $\tau^{2}=i d$. The solutions are given in terms of unitary characters of $\mathrm{G}$.

\section{Introduction}

Let $\mathrm{G}$ be a compact group, let $\sigma, \tau$ be continuous automorphism or continuous anti-automorphism such that $\sigma^{2}=\tau^{2}=i d$. We consider the functional equation

$$
f(x \sigma(y))+f(\tau(y) x)=2 f(x) f(y), \quad x, y \in G,
$$

2010 Mathematics Subject Classification: 39B52, 22C05, 43A30, 22E45

Key words and phrases: functional equation, non-abelian Fourier transform, representation of a compact group 
where $f: G \rightarrow \mathbb{C}$ is the function to determine. This equation, in the case where $\mathrm{G}$ is abelian, has been studied by many authors (see, e.g., Shin'ya [7, Corollary 3.12], and Stetkær [8, Theorem 14.9]). Eq. (1) is a generalization of the following variant of d'Alembert's functional equation

$$
f(x y)+f(\sigma(y) x)=2 f(x) f(y), \quad x, y \in G,
$$

which was introduced and solved on semi-groups by Stetkær in [9]. Some information, applications and numerous references concerning (2), d'Alembert's functional equation

$$
f(x+y)+f(x-y)=2 f(x) f(y), \quad x, y \in \mathbb{R},
$$

and their further generalizations can be found e.g. in ([5, 3, 4, 1, 8, 9, 10, 11]).

The purpose of the present paper is to solve the functional equation (1) in the case where $\mathrm{G}$ is a compact group and possibly non-abelian. Our approach uses the harmonic analysis and the representation theory on compact groups. We note that the idea of using Fourier analysis for solving (1) goes back to $[2]$.

Throughout the rest of this paper, G is a compact group with identity element e. By solutions (resp. representations), we always mean continuous solutions (resp. continuous representations).

\section{Preliminaries}

In this section, we set up some notation and conventions and briefly review some fundamental facts in Fourier analysis which will be used later.

Let $\mathrm{d} x$ denotes the normalized Haar measure on $\mathrm{G}$. Let $\hat{\mathrm{G}}$ stand for the set of equivalence classes irreducible unitary representations of $\mathrm{G}$. For $[\pi] \in \hat{\mathrm{G}}$, the notation $d_{\pi}$ denotes the dimension of the representation space of $\pi$ and $\mathcal{E}_{\pi}=\operatorname{span}\left\{\sqrt{\pi_{i j}}: i, j=1, \ldots, d_{\pi}\right\}$ the linear span of the matrix elements of $\pi$. For $f \in \mathrm{E}^{2}(\mathrm{G})$, the Fourier transform of $\mathrm{f}$ is defined by

$$
\hat{f}(\pi)=\int_{G} f(x) \pi(x)^{-1} d x \in M_{d_{\pi}}(\mathbb{C}) \text { for all }[\pi] \in \hat{G},
$$

where $M_{d_{\pi}}(\mathbb{C})$ is the space of all $d_{\pi} \times d_{\pi}$ complex matrix.

As usual, the left and right regular representations of $G$ in $E^{2}(G)$ are defined by

$$
\left(L_{y} f\right)(x)=f\left(y^{-1} x\right) \text { and }\left(R_{y} f\right)(x)=f(y x)
$$


respectively, where $f \in \mathrm{E}^{2}(\mathrm{G})$ and $x, y \in G$.

The following properties will be useful later

$$
\widehat{\left(L_{y} f\right)}(\pi)=\hat{f}(\pi) \pi(y)^{-1} \text { and } \widehat{\left(R_{y} f\right)}(\pi)=\pi(y) \hat{f}(\pi)
$$

for all $y \in G$, and $\pi \in \hat{G}$.

\section{Main result}

The following Lemmas will be used in the proof of Theorem 1 .

Lemma 1 Let $\mathrm{G}$ be a compact group and $\pi$ be a unitary irreducible representation of $\mathrm{G}$. Suppose every $x \in \mathrm{G}$, there is $\boldsymbol{c}_{x} \in \mathbb{C}$ such that

$$
\pi(\sigma(x))+\pi(\tau(x))=c_{\chi} I_{d_{\pi}},
$$

then $\mathrm{d}_{\pi}=1$.

Proof. Let $(\mathcal{H} ;\langle\rangle$,$) denote the complex Hilbert space on which the represen-$ tation $\pi$ acts. We will consider two cases, $\pi \circ \sigma \simeq \pi \circ \tau$ or not.

In the first case. From (4) we get that

$$
\pi(\sigma(x))_{i j}+\pi(\tau(x))_{i j}=0 \quad \text { for } i \neq j, \quad 1 \leq i, j \leq d_{\pi}, \quad x \in G .
$$

Since $\pi \circ \sigma \not \varkappa \pi \circ \tau$ we have $\mathcal{E}_{\pi \circ \sigma} \perp \mathcal{E}_{\pi \circ \tau}$. Hence $(\pi \circ \sigma)_{i j}=0$ for $i \neq j$, so $\pi \circ \sigma$ is a diagonal matrix. Since $\pi \circ \sigma$ is irreducible we have $\mathrm{d}_{\pi}=1$.

In the second case, i.e., $\pi \circ \sigma \simeq \pi \circ \tau$, there exists a unitary operator $T$ on $\mathcal{H}$ such that

$$
\pi \circ \sigma(x)=\mathrm{T}^{*} \pi \circ \tau(\mathrm{x}) \mathrm{T}, \quad \mathrm{x} \in \mathrm{G} .
$$

Since $\mathrm{T}$ is a unitary matrix, by the spectral theorem for normal operators applied to $\mathrm{T}$, we infer that $\mathrm{T}$ is diagonalizable. Then $\mathcal{H}$ has an orthonormal basis $\left(e_{1}, e_{2}, \ldots, e_{\mathrm{d}_{\pi}}\right)$ consisting of eigenvectors of $\mathrm{T}$. We write $T e_{i}=\lambda_{i} e_{i}$ where $\lambda_{i} \in \mathbb{C}$ for $i=1,2, \ldots, d_{\pi}$. Actually $\left|\lambda_{i}\right|=1$, because $T$ is unitary. For any $i=1,2, \ldots, d_{\pi}$, we compute that

$$
\begin{aligned}
(\pi \circ \sigma(x))_{i i} & =\left\langle\pi \circ \sigma(x) e_{i}, e_{i}\right\rangle=\left\langle(T)^{*} \pi(\tau(x)) e_{i}, e_{i}\right\rangle \\
& =\left\langle\pi(\tau(x)) T e_{i}, T e_{i}\right\rangle=\left\langle\lambda_{i} \pi(\tau(x)) e_{i}, \lambda_{i} e_{i}\right\rangle \\
& =\lambda_{i} \overline{\lambda_{i}}\left\langle\pi(\tau(x)) e_{i}, e_{i}\right\rangle=\left|\lambda_{i}\right|^{2}(\pi \circ \tau(x))_{i i}=(\pi \circ \tau(x))_{i i},
\end{aligned}
$$

for all $x \in \mathrm{G}$. From (4), we infer that

$$
2(\pi \circ \tau(x))_{i i}=2 f(x),
$$


for all $i=1, \ldots, d_{\pi}$ and $x \in G$. Then $d_{\pi}=1$. Indeed, if $d_{\pi}>1$, then (5) implies that $(\pi \circ \tau)_{i i}=(\pi \circ \tau)_{11}$ for all $i=2 \ldots, d_{\pi}$. But if you use Schur's orthogonality relations which say $\frac{1}{\mathrm{~d}_{\pi}}(\pi \circ \tau)_{i i}$ is an orthonormal basis, we get a contradiction. Then $\mathrm{d}_{\pi}=1$.

Lemma 2 Let $\mathrm{f}: \mathrm{G} \rightarrow \mathbb{C}$ be a non-zero solution of (1). Then exists $[\pi] \in \widehat{\mathrm{G}}$ such that $\hat{\mathbf{f}}(\pi)$ is invertible.

Proof. Reformulate (3) to

$$
2 f(x) f=R_{\sigma(x)} f+L_{\tau\left(x^{-1}\right)} f, \quad x \in G .
$$

Taking the Fourier transform to the last equation and using the identities given in section 2 , we have

$$
\hat{\mathrm{f}}(\pi) \pi(\tau(x))+\pi(\sigma(x)) \hat{f}(\pi)=2 \mathrm{f}(x) \hat{f}(\pi), \quad x \in \mathrm{G} .
$$

Since $f \not \equiv 0$, there exists $[\pi] \in \hat{G}$ with $\hat{f}(\pi) \neq 0$. Now, let $v$ be a vector in ker $\hat{f}(\pi)$. From (6), we infer that $\hat{f}(\pi) \pi(\tau(x)) \nu=0$ for all $x \in G$, this implies that $\hat{f}(\pi) \pi(x) v=0$ for all $x \in G$. So $\pi(x) \operatorname{ker} \hat{f}(\pi) \subset \operatorname{ker} \hat{f}(\pi)$ for all $x \in G$. Since $\pi$ is irreducible and $\hat{f}(\pi) \neq 0$, we have $\operatorname{ker} \hat{f}(\pi)=\{0\}$. This implies that $\hat{\mathrm{f}}(\pi)$ is bijective, thus invertible as a matrix.

Lemma 3 Let $\mathrm{f}: \mathrm{G} \rightarrow \mathbb{C}$ be a non-zero solution of (1). Then $\mathrm{f}$ is central.

Proof. Using Lemma 2 and equality (6), we see that there exists $[\pi] \in \hat{\mathrm{G}}$ such that

$$
\pi(\sigma(x))+\hat{f}(\pi)^{-1} \pi(\tau(x)) \hat{f}(\pi)=2 f(x) I_{d_{\pi}}, \quad x \in G .
$$

Taking the trace on both sides of (7) we obtain that

$$
\operatorname{tr}(\pi(\sigma(x)))+\operatorname{tr}(\pi(\tau(x)))=2 d_{\pi} f(x), \quad x \in G,
$$

which abbreviates to

$$
f(x)=\frac{1}{2 d_{\pi}}(\operatorname{tr}(\pi(\sigma(x)))+\operatorname{tr}(\pi(\tau(x)))), \quad x \in G .
$$

Each terms on the right hand side of (8) is a central function, because trace is a central function. Hence $f$ is central.

By help of the previous lemmas, we now describe the complete solution of (1) on an arbitrary compact group. It is clear that $f \equiv 0$ is a solution of (1), so in the following theorem we are only concerned with the non-zero solutions. 
Theorem 1 The non-zero solutions $\mathrm{f}: \mathrm{G} \rightarrow \mathbb{C}$ of (1) are the functions of the form $\mathrm{f}=(\chi+\chi \circ \sigma \circ \tau) / 2$, where $\chi: \mathrm{G} \rightarrow \mathbb{C}$ is a character such that:

1. $\chi \circ \sigma \circ \tau=\chi \circ \tau \circ \sigma$, and

2. $\chi$ is $\sigma$-even and/or $\tau$-even (i.e., $\chi \circ \sigma=\chi$ and/or $\chi \circ \tau=\chi$ ).

Proof. We have $f$ is central. This implies that $\hat{f}(\pi)$ is an intertwining operator for $\pi$. But $\pi$ is irreducible, so $\hat{f}(\pi)=\mu \mathrm{I}_{\mathrm{d}_{\pi}}$ for some $\mu \in \mathbb{C}$ by Schur's lemma.

Actually $\mu \neq 0$, because $\hat{f}(\pi) \neq 0$. Now Eq. (7) coalesce into

$$
\pi(\sigma(x))+\pi(\tau(x))=2 f(x) I_{d_{\pi}}, \quad x \in G .
$$

From $\mathrm{d}_{\pi}=1$, we see that $\pi$ is a unitary character, say $\pi=\chi$, so

$$
f=\frac{\chi \circ \sigma+\chi \circ \tau}{2}
$$

If $\chi \circ \sigma=\chi \circ \tau$, then letting $\chi:=\chi \circ \sigma$ we have $f=\chi$. Substituting $f=\chi$ into (1) we get that $\chi \circ \sigma+\chi \circ \tau=2 \chi$. So $\chi=\chi \circ \sigma=\chi \circ \tau$. Then $f$ has the desired form.

If $\chi \circ \sigma \neq \chi \circ \tau$, substituting $f=(\chi \circ \sigma+\chi \circ \tau) / 2$ into (1) we find after a reduction that

$$
\begin{aligned}
& \chi \circ \sigma(x)[x(y)+\chi \circ \sigma \circ \tau(y)-\chi \circ \sigma(y)-\chi \circ \tau(y)]+\chi \circ \tau(x)[x \circ \tau \circ \sigma(y) \\
& +\chi \circ \tau \circ \tau(y)-\chi \circ \sigma(y)-\chi \circ \tau(y)]=0
\end{aligned}
$$

for all $x, y \in G$. Since $\chi \circ \sigma \neq \chi \circ \tau$ we get from the theory of multiplicative functions (see for instance [9, Theorem 3.18]) that both terms are 0, so

$$
\left\{\begin{array}{l}
x \circ \sigma(x)[x(y)+\chi \circ \sigma \circ \tau(y)-x \circ \sigma(y)-\chi \circ \tau(y)]=0 \\
x \circ \tau(x)[x \circ \tau \circ \sigma(y)+\chi(y)-\chi \circ \sigma(y)-\chi \circ \tau(y)]=0
\end{array}\right.
$$

for all $x, y \in G$. Since $\chi \circ \sigma \neq \chi \circ \tau$ at least one of $\chi \circ \sigma$ and $\chi \circ \tau$ is not zero. We have $\chi \circ \sigma \neq 0$ and $\chi \circ \tau \neq 0$. From (1), we have

$$
\chi \circ \sigma+\chi \circ \tau=\chi+\chi \circ \sigma \circ \tau=\chi \circ \tau \circ \sigma+\chi .
$$

Using $\chi+\chi \circ \sigma \circ \tau=\chi \circ \tau \circ \sigma+\chi$ and the fact that $\chi \circ \sigma \neq \chi \circ \tau$, we see that $\chi=\chi$ and $\chi \circ \sigma \circ \tau=\chi \circ \tau \circ \sigma$. Thus

$$
\chi \circ \tau=\chi \circ \sigma \circ \tau \circ \sigma .
$$


We now use $\chi \circ \sigma+\chi \circ \tau=\chi+\chi \circ \sigma \circ \tau$, we get that $\chi$ is $\sigma$-even or $\tau$-even.

Finally, in view of these cases we deduce that $f$ has the form stated in Theorem 1.

Similarly to Theorem 1, we can get the solution of functional equation (1) when $\sigma, \tau$ are continuous anti-automorphism such that $\sigma^{2}=\tau^{2}=i d$.

Theorem 2 The non-zero solutions $\mathrm{f}: \mathrm{G} \rightarrow \mathbb{C}$ of (1) are the functions of the form $\mathrm{f}=(\chi+\chi \circ \sigma \circ \tau) / 2$, where $\chi: \mathrm{G} \rightarrow \mathbb{C}$ is a character such that:

1. $\chi \circ \sigma \circ \tau=\chi \circ \tau \circ \sigma$, and

2. $\chi$ is $\sigma$-even and/or $\tau$-even (i.e., $\chi \circ \sigma=\chi$ and/or $\chi \circ \tau=\chi$ ).

Proof. The proof is similar to the proof of Theorem 1.

\section{Some applications of the main result}

As immediate consequences of Theorems 1 and 2, we have the following corollaries.

Corollary 1 Let $\mathrm{G}$ be a compact group and $\sigma$ be a continuous homomorphism or continuous anti-homomorphism such that $\sigma \circ \sigma=i d$. The non-zero solutions $f: G \rightarrow \mathbb{C}$ of of the functional equation

$$
f(x \sigma(y))+f(\sigma(y) x)=2 f(x) f(y), \quad x, y \in G,
$$

are the functions of the form $\mathrm{f}=\mathrm{\chi}$, where $\mathrm{\chi}: \mathrm{G} \rightarrow \mathbb{C}$ is a character such that $\chi$ is $\sigma$-even.

Proof. It suffices to take $\sigma(x)=\tau(x)$ for all $x \in G$ in Theorem 1 or in Theorem 2 .

Corollary 2 Let $\mathrm{G}$ be a compact group and $\sigma$ be a continuous homomorphism such that $\sigma \circ \sigma=i d$. The non-zero solutions $f: G \rightarrow \mathbb{C}$ of of the functional equation

$$
f(x \sigma(y))+f(y x)=2 f(x) f(y), \quad x, y \in G,
$$

are the functions of the form $\mathrm{f}=(\chi+\chi \circ \sigma) / 2$, where $\chi: G \rightarrow \mathbb{C}$ is a character.

Proof. It suffices to take $\tau(x)=x$ for all $x \in G$ in Theorem 1 . 
Corollary 3 Let $\mathrm{G}$ be a compact group and $\tau$ be a continuous homomorphism such that $\tau \circ \tau=i d$. The non-zero solutions $f: G \rightarrow \mathbb{C}$ of of the functional equation

$$
f(x y)+f(\tau(y) x)=2 f(x) f(y), \quad x, y \in G
$$

are the functions of the form $\mathrm{f}=(\chi+\chi \circ \tau) / 2$, where $\mathrm{\chi}: \mathrm{G} \rightarrow \mathbb{C}$ is a character.

Proof. It suffices to take $\sigma(x)=x$ for all $x \in \mathrm{G}$ in Theorem 1 .

Corollary 4 Let $\mathrm{G}$ be a compact group and $\sigma$ be a continuous anti-homomorphism such that $\sigma \circ \sigma=i d$. The non-zero solutions $f: G \rightarrow \mathbb{C}$ of of the functional equation

$$
f(x \sigma(y))+f\left(y^{-1} x\right)=2 f(x) f(y), \quad x, y \in G,
$$

are the functions of the form $\mathrm{f}=(\chi+\bar{\chi} \circ \sigma) / 2$, where $\mathrm{X}: \mathrm{G} \rightarrow \mathbb{C}$ is a character such that $\chi$ is $\sigma$-even and/or $\bar{\chi}=\chi$.

Proof. It suffices to take $\tau(x)=x^{-1}$ for all $x \in G$ in Theorem 2 .

Corollary 5 Let $\mathrm{G}$ be a compact group and $\tau$ be a continuous anti-homomorphism such that $\tau \circ \tau=i d$. The non-zero solutions $f: G \rightarrow \mathbb{C}$ of of the functional equation

$$
f\left(x y^{-1}\right)+f(\tau(y) x)=2 f(x) f(y), \quad x, y \in G,
$$

are the functions of the form $\mathrm{f}=(\chi+\overline{\chi \circ \tau}) / 2$, where $\chi: G \rightarrow \mathbb{C}$ is a character such that $\chi$ is $\tau$-even and/or $\bar{\chi}=\chi$.

Proof. It suffices to take $\sigma(x)=x^{-1}$ for all $x \in G$ in Theorem 2 .

Corollary 6 The non-zero solutions $f: G \rightarrow \mathbb{C}$ of the functional equation

$$
f(x y)+f(y x)=2 f(x) f(y), x, y \in G,
$$

are the functions of the form $\mathrm{f}=\chi, \mathrm{x}$, whe $\mathrm{\chi}: \mathrm{G} \rightarrow \mathbb{C}$ is a unitary character.

Proof. It suffices to take $\sigma(x)=\tau(x)=x$ for all $x \in G$ in Theorem 1 .

Corollary 7 The non-zero solutions $\mathrm{f}: \mathrm{G} \rightarrow \mathbb{C}$ of the functional equation

$$
f\left(x y^{-1}\right)+f\left(y^{-1} x\right)=2 f(x) f(y), x, y \in G,
$$

are the functions of the form $\mathrm{f}=\chi$, where $\mathrm{\chi}: \mathrm{G} \rightarrow \mathbb{C}$ is a unitary character such that $\bar{\chi}=\chi$.

Proof. It suffices to take $\sigma(x)=\tau(x)=x^{-1}$ for all $x \in G$ in Theorem 2 . 


\section{References}

[1] A. Chahbi, B. Fadli, S. Kabbaj,A generalization of the symmetrized multiplicative Cauchy equation, Acta Math. Hung., 149 (2016), 170-176.

[2] W. Chojnacki, On some functional equation generalizing Cauchy's and d'Alembert's functional equations, Colloq. Math., 55 (1988), 169-178.

[3] W. Chojnacki,On group decompositions of bounded cosine sequences, Studia Math., 181 (2007), 61-85.

[4] W. Chojnacki, On uniformly bounded spherical functions in Hilbert space, Aequationes Math., 81 (2011), 135-154.

[5] Iz. EL-Fassi, A. Chahbi, S. Kabbaj, The Solution of a class functional equations on semi-groups, Filomat, to appear.

[6] G. Folland, A Course in Abstract Harmonic Analysis, CRC Press, Boca Raton, FL, 1995.

[7] H. Shin'ya, Spherical matrix functions and Banach representability for locally compact motion groups, Japan. J. Math. (N.S.), 28 (2002), 163-201.

[8] H. Stetkær, Functional Equations on Groups, World Scientfic, Singapore, 2013.

[9] H. Stetkær,A variant of d'Alemberts functional equation, Aequationes Math., 89 (2015), 657-662.

[10] L. Székelyhidi,D'Alemberts functional equation on compact groups, $B a$ nach J. Math. Anal., 1 (2) (2007), 221-226.

[11] D. Yang,Functional Equations and Fourier Analysis, Canad. Math. Bull., 56 (2013), 218-224. 